\title{
Fluorescence Enhancement Factors on Optical Antennas: Enlarging the Experimental Values without Changing the Antenna Design
}

\author{
Jérôme Wenger \\ Institut Fresnel, CNRS, Aix Marseille Université, Ecole Centrale Marseille, Campus St. Jérôme, 13013 Marseille, France \\ Correspondence should be addressed to Jérôme Wenger, jerome.wenger@fresnel.fr \\ Received 13 July 2011; Accepted 3 October 2011 \\ Academic Editor: A. Femius Koenderink \\ Copyright (C) 2012 Jérôme Wenger. This is an open access article distributed under the Creative Commons Attribution License, \\ which permits unrestricted use, distribution, and reproduction in any medium, provided the original work is properly cited.

\begin{abstract}
Plasmonic antennas offer promising opportunities to control the emission of quantum objects. As a consequence, the fluorescence enhancement factor is widely used as a figure of merit for a practical antenna realization. However, the fluorescence enhancement factor is not an intrinsic property of the antenna. It critically depends on several parameters, some of which are often disregarded. In this contribution, I explore the influence of the setup collection efficiency, emitter's quantum yield, and excitation intensity. Improperly setting these parameters may significantly alter the enhancement values, leading to potential misinterpretations. The discussion is illustrated by an antenna example of a nanoaperture surrounded by plasmonic corrugations.
\end{abstract}

\section{Introduction}

Plasmonic antennas are receiving a large interest to interface light with nanoscale quantum emitters on dimensions much beyond the optical wavelength $[1,2]$. Recent developments involve squeezing light into nanoscale volumes [3], enhancing the excitation and emission rate of individual emitters [4-8], tuning the luminescence spectrum $[9,10]$, polarization [11], and directivity properties [12-16]. Several plasmonic systems are being investigated to enhance the luminescence emission of fluorescent molecules or quantum dots, such as metallic nanoparticles $[4,5,17-20]$, core-shell particles [21], thin films [22, 23], nanoantennas $[6,7,15$, 24], nanowires [16], nanoporous gold [25], nanopockets [26], metallic gratings [27], nanoaperture arrays [28], and single nanoapertures $[29,30]$. A general review on surfaceenhanced fluorescence can be found in [31].

A natural question while performing experiments on nanoantenna-enhanced luminescence deals with the quantification of the luminescence enhancement factor $\eta_{F}$, which is commonly defined as the ratio of the detected radiation power per emitter with the antenna to the reference radiation power per emitter without the antenna. $\eta_{F}$ determines how many extra photons are detected for each emitter thanks to the use of the optical antenna. It is well known that this factor critically depends on several parameters: the antenna material and geometry, its spectral resonance, and overlap with the emitter's absorption and luminescence spectra, as well as the emitter's orientation and location respective to the antenna [32]. These many parameters often hide the influence of other parameters: the collection efficiency used in the experiments, the emitter's quantum yield in the absence of the antenna, and the excitation intensity respective to the saturation process. These last three parameters are more technically oriented and depend on the specific experimental implementation. Therefore, they have received less attention from theoretical investigations. However, as I will show below, these parameters have a major influence on the measured values of the luminescence enhancement factor. Improperly setting these parameters may significantly alter the value found for the luminescence enhancement factor, leading to potential experimental pitfalls.

In this contribution, I explore the influence of the collection efficiency, molecular quantum yield, and excitation intensity on the fluorescence enhancement factor. Several rules are derived to maximize the enhancement factor by tuning the experimental conditions without affecting the antenna's design. Using the fluorescence enhancement factor 


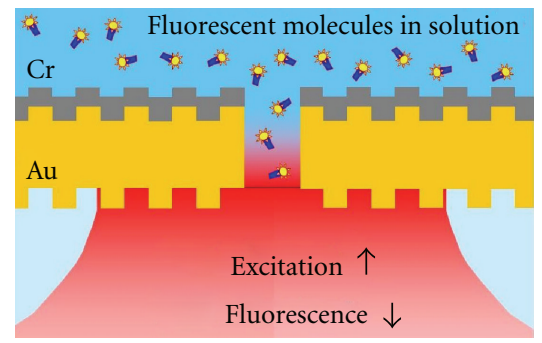

(a)

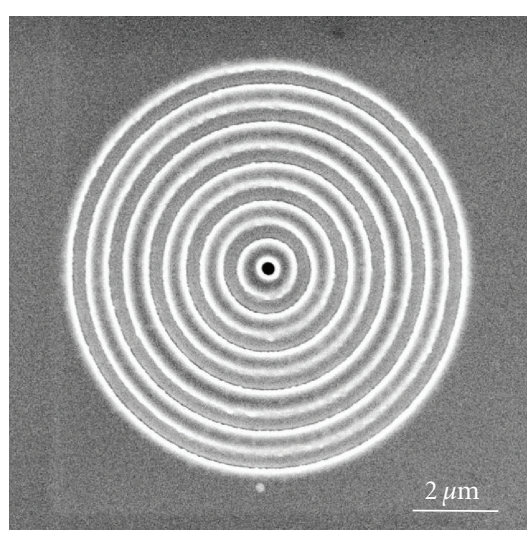

(b)

FIgURE 1: Corrugated aperture antenna used here to illustrate the influence of experimental parameters on the measured fluorescence enhancement factor. (a) Experimental configuration to probe molecules randomly diffusing in aqueous solution. (b) Scanning electron microscope image of the antenna with five corrugations. Adapted with permission from [33]. Copyright 2011 American Chemical Society.

as a figure of merit to compare between different antenna designs has to be done with caution. Analytical formulas are illustrated by a practical antenna example made of a single nanoaperture surrounded by five shallow grooves in a gold film (Figure 1). This optical antenna can significantly enhance the fluorescence count rates per molecule and control the emission directivity, as demonstrated recently [33]. Very much in the spirit of the review in [34] about the comparison between experiments and numerical simulations, I hope that this paper may initiate some reflection and avoid any misleading interpretation.

\section{Designing the Experiment to Maximize the Fluorescence Enhancement Factor}

A loophole in any measurement of the luminescence enhancement factor deals with the normalization of the detected signal per emitter. This can be done by performing experiments on single molecules directly $[4,5,7]$, by surface normalization $[25,28]$, or by calibrating the number of emitters with fluorescence correlation spectroscopy $[29,30]$. Hereafter, I consider that this normalization issue is resolved and that a reliable value for the detected radiated power per emitter can be obtained. I also assume that the signal per emitter has been averaged over several positions and dipole orientations. The aim of this paper is to focus on the influence of experimental parameters on the measured values for the fluorescence enhancement factor $\eta_{F}$.

2.1. Theoretical Background: Fluorescence Count Rate per Molecule. Throughout this paper, the quantum emitter is modelled by a two energy levels system. $k_{r}$ and $k_{n r}$ are the rate constants for radiative and nonradiative transitions from the excited singlet state to the ground state. The total deexcitation rate from the excited singlet state is noted as $k_{\text {tot }}=$ $k_{r}+k_{n r}$, which is the inverse of the excited state lifetime $\tau$. $\phi=k_{r} / k_{\text {tot }}=k_{r} /\left(k_{r}+k_{n r}\right)$ is the quantum yield. $\sigma I_{e}$ is the excitation rate, where $\sigma$ denotes the excitation cross-section and $I_{e}$ the excitation intensity. Under steady-state conditions, the fluorescence count rate per molecule CRM is given by [35]

$$
\mathrm{CRM}=\kappa \phi \frac{\sigma I_{e}}{1+I_{e} / I_{s}} .
$$

We note $\kappa$ the light collection efficiency and $I_{s}=k_{\text {tot }} / \sigma$ the saturation intensity.

Equation (1) takes two limits for the extreme regimes of weak excitation $\left(I_{e} \ll I_{s}\right)$ and fluorescence saturation $\left(I_{e} \gg\right.$ $\left.I_{s}\right)$. In the weak excitation regime, the CRM reduces to

$$
\mathrm{CRM}=\kappa \phi \sigma I_{e} \quad\left(I_{e} \ll I_{s}\right)
$$

which indicates that the fluorescence rate per molecule is proportional to the collection efficiency, the quantum yield, and the excitation intensity. This expression appears to be the one commonly used in fluorescence spectroscopy and microscopy applied to the life sciences [36].

In the saturation regime $I_{e} \gg I_{s}$, (1) reduces to

$$
\mathrm{CRM}=\kappa \phi \sigma I_{s}=\kappa k_{r} \quad\left(I_{e} \gg I_{s}\right)
$$

which indicates that the fluorescence rate per molecule at saturation is determined only by the radiative rate and the collection efficiency and is of course independent on the excitation rate. This expression is generally used for singlephoton sources used for quantum communication purposes, such as quantum cryptography $[37,38]$.

2.2. Excitation Intensity. From (1), it is apparent that the detected fluorescence rate per emitter-hence the fluorescence enhancement factor-bears a complex dependence upon the excitation intensity $I_{e}$. In the weak excitation regime (below the transition to saturation), the CRM is linearly proportional with $I_{e}$, and the fluorescence enhancement factor $\eta_{F}$ can therefore be expressed as

$$
\eta_{F}=\frac{\mathrm{CRM}^{*}}{\mathrm{CRM}}=\frac{\kappa^{*}}{\kappa} \frac{\phi^{*}}{\phi} \frac{I_{e}^{*}}{I_{e}} \quad\left(I_{e} \ll I_{s}\right) .
$$




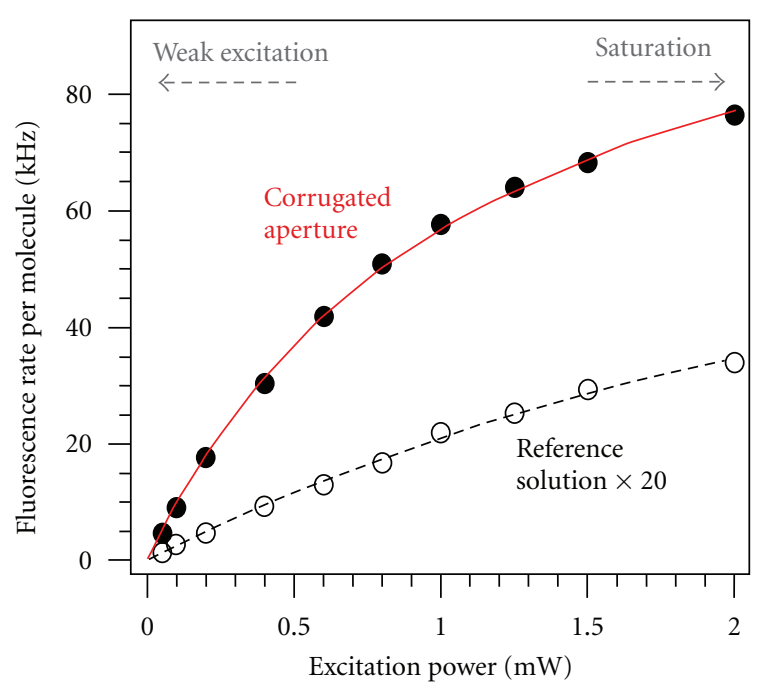

(a)

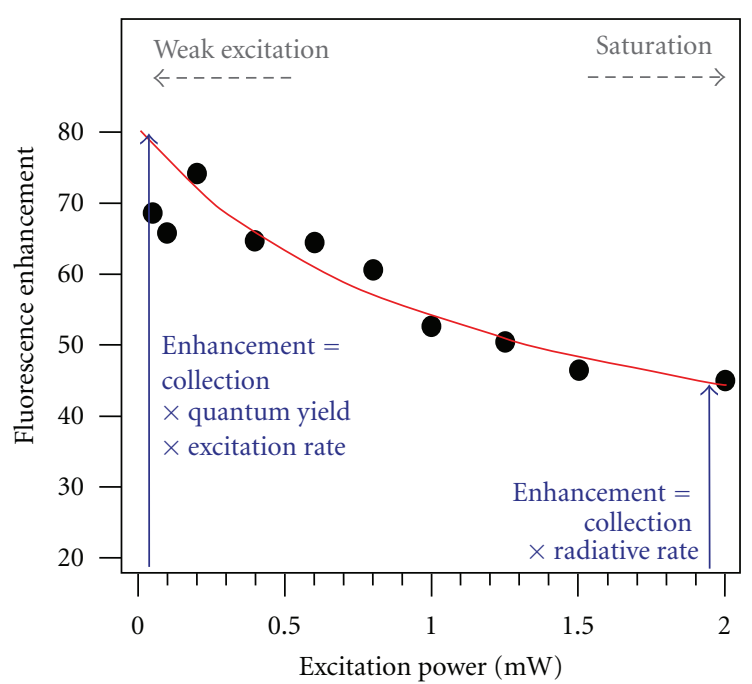

(b)

Figure 2: (a) Average fluorescence count rate per molecule detected in the case of the corrugated aperture presented in Figure 1 (filled circles) and the reference solution (empty circles). The molecules have a quantum yield in solution of 30\% (Alexa Fluor 647), and the numerical aperture used for both excitation and collection is 0.5. (b) Fluorescence enhancement factor for increasing excitation power. The arrows indicate the two extreme cases of weak excitation $\left(I_{e} \ll I_{s}\right)$ and saturation $\left(I_{e} \gg I_{s}\right)$. Figure adapted with permission from [33].

The superscript * denotes the presence of the antenna. In the weak excitation regime, the fluorescence enhancement factor is the product of the enhancements in the collection efficiency, the quantum yield, and the excitation intensity (here we assume that the nanoantenna does not modify significantly the fluorophore's absorption cross section: $\sigma^{*}=$ $\sigma)$.

Equation (4) can be rewritten in a slightly different manner to introduce the gains in the radiative rate $k_{r}^{*} / k_{r}$ and the total fluorescence lifetime reduction $k_{\mathrm{tot}}^{*} / k_{\mathrm{tot}}$ :

$$
\eta_{F}=\frac{\kappa^{*}}{\kappa} \frac{k_{r}^{*}}{k_{r}} \frac{k_{\mathrm{tot}}}{k_{\mathrm{tot}}^{*}} \frac{I_{e}^{*}}{I_{e}}=\frac{\kappa^{*}}{\kappa} \frac{k_{r}^{*}}{k_{r}} \frac{\tau^{*}}{\tau} \frac{I_{e}^{*}}{I_{e}} \quad\left(I_{e} \ll I_{s}\right)
$$

This equation shows that the fluorescence enhancement factor is proportional to the gains in the radiative rates $k_{r}$ and inversely proportional to the total deexcitation rates $k_{\text {tot }}$. Since $k_{\mathrm{tot}}^{*} / k_{\mathrm{tot}}=\tau / \tau^{*}$ is also the reduction in the fluorescence lifetimes, a strong reduction in the fluorescence lifetimes (sometimes also referred to as Purcell factor) is actually detrimental to the fluorescence enhancement factor. Clearly, the fluorescence lifetimes reduction (Purcell factor) must not be confused with the fluorescence count rate enhancement (see also the discussion in [39]). Observing a strong lifetime reduction due to the nanoantenna can be related to an increase in the nonradiative transition rate $k_{n r}^{*}$, mostly related to ohmic losses. This is the so-called quenching effect, which is certainly not related to any increase in the fluorescence count rate per emitter.

In the saturation regime, it is evident from (3) that the fluorescence enhancement factor at saturation depends only on the gains in collection efficiency and radiative rate:

$$
\eta_{F}=\frac{\kappa^{*}}{\kappa} \frac{k_{r}^{*}}{k_{r}} \quad\left(I_{e} \gg I_{s}\right)
$$

Interestingly, at saturation of the fluorescence process, the fluorescence enhancement is found independent on the nonradiative transition rate $k_{n r}$, which means that metal quenching might not be an issue in this particular configuration. At saturation, the sole figure of merit is the ability of the emitter-nanoantenna system to radiate photons into the photonic modes used for detection, which is quantified by $\kappa k_{r}$ (this rate is sometimes written $k_{e m}=\kappa k_{r}[30,33]$ ). Comparing the enhancement $\eta_{F}$ in the saturation regime to the case of weak excitation, it turns out that $\eta_{F}$ in the weak excitation regime is larger by a factor $\left(I_{e}^{*} / I_{e}\right)\left(\tau^{*} / \tau\right)$, which amounts to the gain in the excitation intensity divided by the fluorescence lifetime reduction. Depending on the interplay between excitation enhancement and quenching losses, high fluorescence enhancement values can be preferentially reached by working in the weak excitation regime (most common cases) or at fluorescence saturation (in the case of strong quenching losses). However, it must be kept in mind that the saturation intensity increases with the reduction of the fluorescence lifetime. Therefore reaching the saturation regime will require more excitation power in the case of strong quenching, that may even induce photodamage to the molecule or to the structure.

Figure 2 illustrates the dependence of the fluorescence enhancement factor on the excitation intensity, in the example case of the corrugated aperture displayed in Figure 1. The fluorescence count rates per molecule in the case of the antenna and the reference solution are presented in Figure 2(a) versus the excitation power, together with numerical fits according to the general expression (1) (the CRM for the reference solution has been multiplied by an arbitrary 20x factor to ease viewing). A transition between the regimes of weak excitation and saturation is clearly seen for excitation powers around $1 \mathrm{~mW}$ (excitation made 
through a 0.5 NA objective, so the power to reach saturation is significantly higher as compared to focusing with a high NA objective). The transition towards saturation can be quantified by the saturation intensity $I_{s}$. For the experiments in Figure 2(a), we found $I_{s}^{*}=1.2 \mathrm{~mW}$ for the antenna and $I_{s}=3.4 \mathrm{~mW}$ for the reference solution [33] (in the case of a 1.2 NA objective, the saturation intensities would be $I_{s}^{*}=$ $130 \mu \mathrm{W}$ for the antenna and $I_{s}=510 \mu \mathrm{W}$ for the reference solution).

Changing the excitation intensity has a strong influence on the fluorescence enhancement factor, as seen in Figure 2(b). In the weak excitation regime, the enhancement is maximum $(\sim 80 \times$ for this example), while it decreases to $\sim 40 \times$ when saturation is reached. This can be directly explained by the different contributions of the gains in radiative rate, quantum yield, and excitation intensity. $\eta_{F}$ in the weak excitation regime is larger by a factor $\left(I_{e}^{*} / I_{e}\right)\left(\tau^{*} / \tau\right) \simeq 2$, which corresponds well to the gain in the excitation intensity $(\approx 5.5)$ divided by the fluorescence lifetime reduction $(\approx 2)$ as measured separately [33].

\subsection{Emitter's Reference Quantum Yield without the Antenna.} In this section, we take a closer look at the influence of the emitter's quantum yield $\phi=k_{r} /\left(k_{r}+k_{n r}\right)$ on the fluorescence enhancement factor. The basic question is how shall we choose $\phi$ for the reference solution (without the antenna) so as to maximize the fluorescence enhancement? Hereafter, the excitation intensity is set to the weak excitation regime $I_{e} \ll$ $I_{s}$ (in the saturation regime, the fluorescence enhancement does not depend directly on $\phi$, so the discussion is useless).

With the nanoantenna, the quantum yield is modified to $\phi^{*}=k_{r}^{*} /\left(k_{r}^{*}+k_{n r}+k_{\mathrm{abs}}^{*}\right)$, where a new nonradiative decay route $k_{\mathrm{abs}}^{*}$ is introduced to take into account the ohmic losses into the metal and nonradiative energy transfers to the free electrons in the metal. It is also assumed that the nonradiative rate $k_{n r}$ is not affected by the antenna. After some basic algebra, (4) can be rewritten:

$$
\eta_{F}=\frac{\kappa^{*}}{\kappa} \frac{k_{r}^{*}}{k_{r}} \frac{I_{e}^{*}}{I_{e}} \frac{1}{(1-\phi)+\phi \zeta}
$$

with $\zeta=\left(k_{r}^{*}+k_{\mathrm{abs}}^{*}\right) / k_{r}$. In the limit of a "poor" emitter $\phi \ll 1$ and $\phi \zeta \ll 1,(7)$ resumes to

$$
\eta_{F}=\frac{\kappa^{*}}{\kappa} \frac{k_{r}^{*}}{k_{r}} \frac{I_{e}^{*}}{I_{e}} \quad(\phi \ll 1)
$$

which is the product of the gains in collection efficiency, radiative rate, and excitation intensity (this value can also be seen as the enhancement factor found at saturation times the gain in excitation intensity).

In the case of a perfect emitter $\phi \simeq 1,(7)$ resumes to

$$
\eta_{F}=\frac{\kappa^{*}}{\kappa} \frac{I_{e}^{*}}{I_{e}} \quad(\phi \simeq 1)
$$

if we assume that $k_{r}^{*} \gg k_{\text {abs }}^{*}$, meaning that the antenna has a large efficiency. Thus for a perfect emitter, the fluorescence enhancement at weak excitation intensity is given by the product of the gains in collection efficiency and excitation

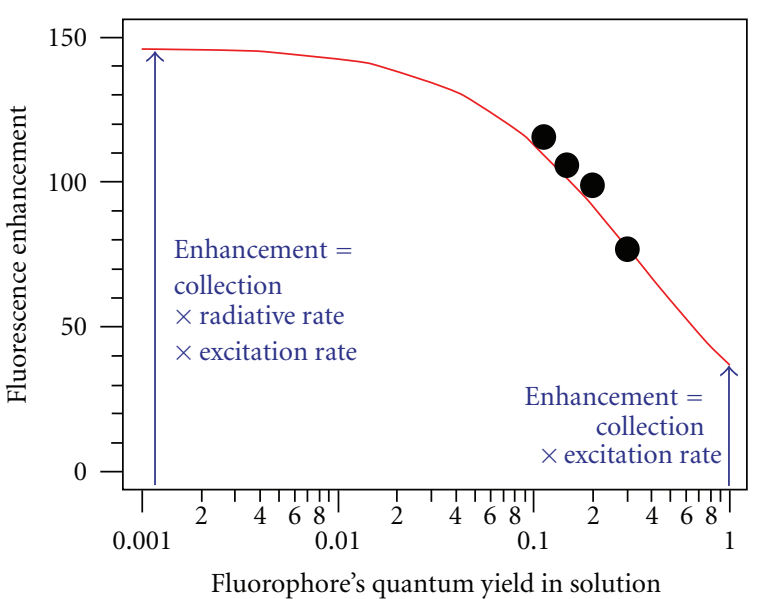

FIGURE 3: Fluorescence enhancement factor versus the dye's quantum yield $\phi$ in solution (taken without the antenna), in the case of weak excitation. The arrows indicate the two extreme cases of perfect dye $(\phi=1)$ or poor emitter $(\phi \ll 1)$. The experimental data is taken from [33].

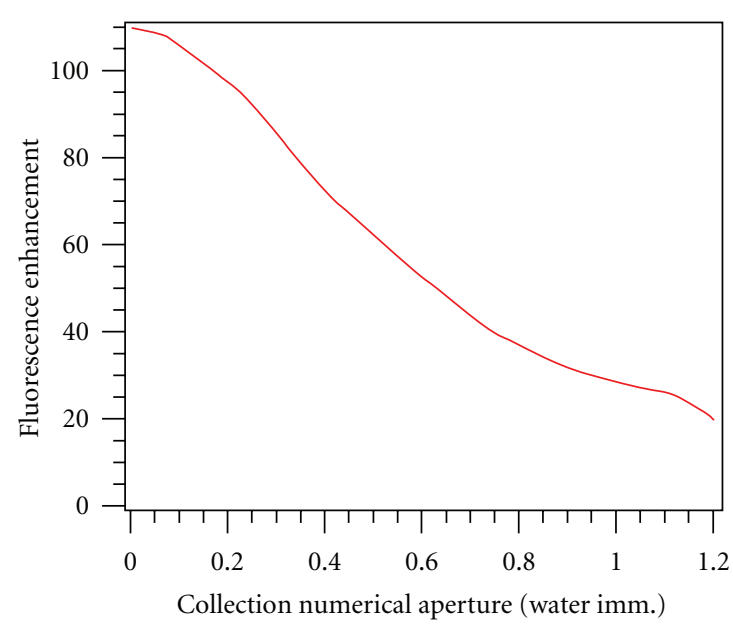

FIGURE 4: Influence of the numerical aperture used for collection on the fluorescence enhancement factor. Computation derived from the experimental data published in [33], in the case of weak excitation.

intensity. The fluorescence enhancement in the case of a poor emitter is larger by a factor $k_{r}^{*} / k_{r}$, indicating that, in order to maximize the value for $\eta_{F}$, one should preferentially select emitters with rather low quantum yields (as long as the experimental signal-to-noise ratio is sufficiently high to provide for reliable measurements).

Figure 3 illustrates this discussion, based on the modification of the fluorescence properties calibrated in [33]. $\eta_{F}$ grows as the reference quantum yield $\phi$ is decreased, up to a plateau for $\phi<0.02$. Again, the maximum value for the fluorescence enhancement in this example can be significantly different if a perfect dye is used $\eta_{F} \approx 35$ or if a poor emitter is chosen $\eta_{F} \approx 150$. 


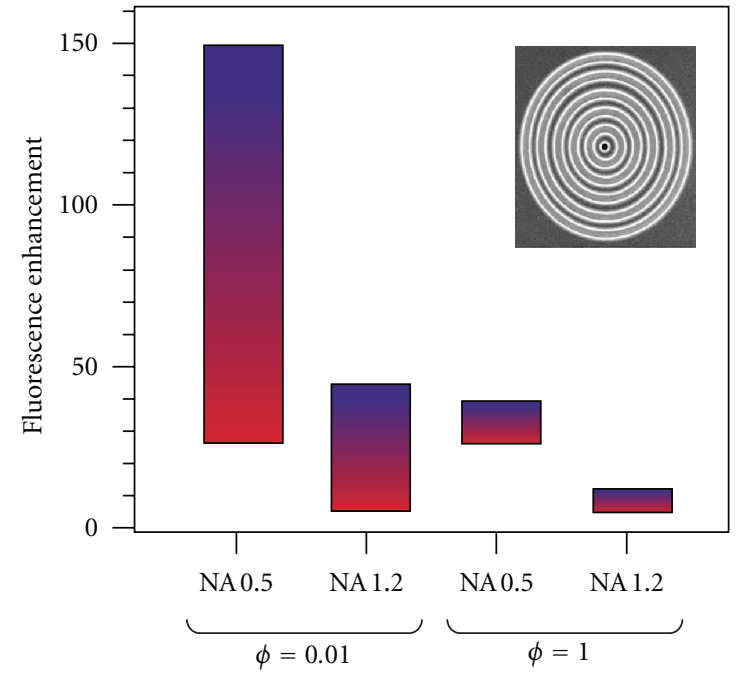

(a)

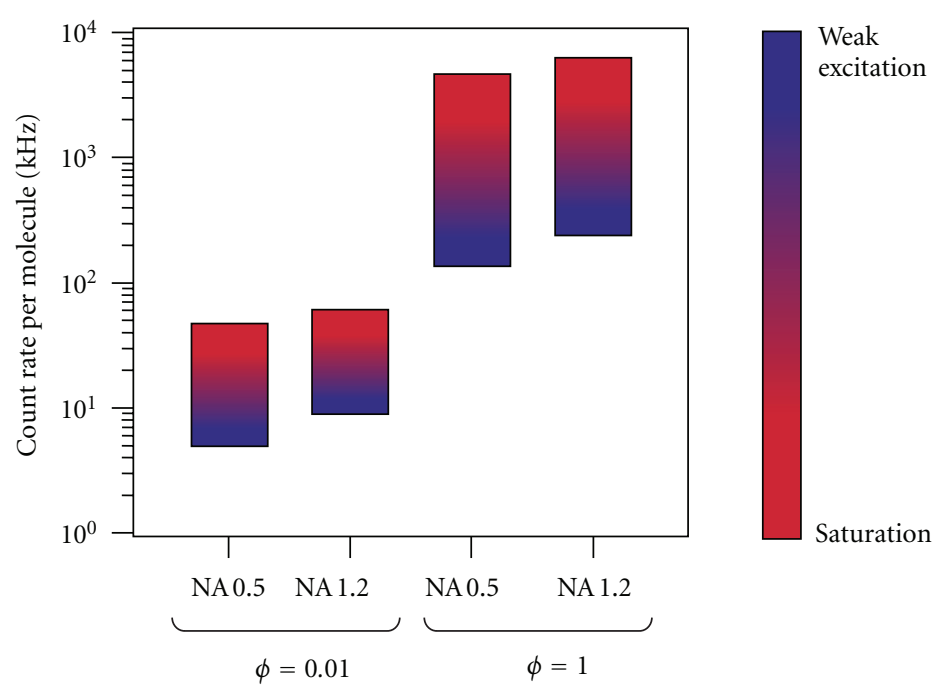

(b)

FIGURE 5: Fluorescence enhancement factor (a) and fluorescence detection rate per emitter (b) for different numerical apertures (NAs) used for collection, different quantum yield $\phi$ of the emitter in solution (in the absence of the antenna) and in the limit of weak excitation (blue) or fluorescence saturation (red). The antenna used here is the corrugated aperture with five grooves described in Figure 1 and calibrated in [33].

2.4. Luminescence Collection Efficiency. All the formulas presented here for the fluorescence enhancement factor are proportional to the gain in collection efficiency $\kappa^{*} / \kappa$, independently on the choice for excitation intensity or quantum yield. This is a direct consequence that obviously only the photons that are collected by the setup contribute to the detected fluorescence. Therefore, to maximize the fluorescence enhancement factor, one has to maximize the collection efficiency gain $\kappa^{*} / \kappa$. This can be done by tuning the antenna so as to maximize the directivity $[15,40,41]$ and by minimizing the collection efficiency $\kappa$ used for the reference. In other words, to maximize $\eta_{F}$, an objective with low numerical aperture should be used in accordance with the peak angular emission of the antenna's radiation pattern.

Figure 4 displays the influence of the numerical aperture used for fluorescence collection on the detected fluorescence enhancement factor. The antenna used here is again the corrugated aperture presented in Figure 1. It was found that the fluorescence radiation pattern with the antenna presented a peak in the direction normal to the sample plane with an angular divergence of $\pm 15^{\circ}$. Figure 4 presents the computed fluorescence enhancement factor integrated over the whole collection NA. Minimizing the collection NA to values below 0.1 maximizes the fluorescence enhancement factor up to values $\sim 110$, whereas a large collection NA of 1.2 reduces the effective enhancement factor to $\sim 20$ as an effect of angular averaging.

\section{Discussion}

From the aforementioned investigations on the role of the different experimental parameters on the measured fluorescence enhancement factor, a general "rule of thumb" can be derived: the lower the reference without the antenna, the higher the enhancement factor. In other words, to obtain high enhancement factors, one should preferentially select a weak excitation regime, a dye with low quantum yield and a low collection NA. Of course, this comes at the expense of lower signal (at least for the reference case) and higher noise.

To illustrate the broad range of fluorescence enhancement factors that can be measured using the same nanoantenna design, different computations have been performed using the antenna presented in Figure 1 with either 0.5 or 1.2 NA microscope objective, 0.01 or 1 quantum efficiencies and in the limits of weak excitation or fluorescence saturation. The results are graphically presented in Figure 5(a). In the extreme case of low reference without the antenna (0.5 NA, $\phi=0.01$, weak excitation), the fluorescence enhancement is maximum $\sim 150$, while it is minimum $\sim 6$ when the reference is the highest (1.2 NA, $\phi=1$, saturation).

This clearly shows that the fluorescence enhancement factor is not an absolute figure of merit for a given nanoantenna. This is in strong opposition with the directivity, which is another figure of merit commonly used to characterize the quality of an antenna design and which can be intrinsic to the antenna (if properly measured $[2,33,41]$ ).

The fluorescence enhancement factor is an intuitive metric that is commonly used in surface-enhanced (or metalenhanced) fluorescence. The experimental setup can be adequately tuned so as to maximize the measured values of $\eta_{F}$. However, it should be kept in mind that the final goal in the detection of molecules or the generation of single photons is to realize bright sources out of single quantum emitters [42]. Thus the true figure of merit is not the enhancement factor ("how much do we gain") but instead the fluorescence count rate per emitter ("how much do we 
have"). High enhancement factors should not be confused with bright sources. To illustrate this, Figure 5(b) displays the detected count rate per molecule corresponding to the cases in Figure 5(a). To maximize the detection rate, one has to select the conditions of high collection NA, high quantum yield, and high excitation intensity (saturation). These conditions correspond to the ones leading to the minimum fluorescence enhancement factor in Figure 5(a). Reciprocally, the conditions found for maximum enhancement lead to the minimum detection rates. Taking a low emission for reference and enhancing it (a lot) do not necessarily compensate for the low detection rate to start with. Of course, all this discussion strongly depends on the final application of the nanoantenna-emitter system and the initial photophysical properties of the emitter.

\section{Conclusion}

This paper has explored the influence of several parameters on the fluorescence enhancement factor with an optical antenna: excitation intensity, emitter's quantum yield, and collection efficiency. General rules have been derived to obtain high enhancement factors by tuning the experimental conditions without affecting the antenna's design. Experimental conditions leading to a low reference signal without the antenna should be preferentially selected to maximize $\eta_{F}$. This corresponds to weak excitation regime, dye with low quantum yield and low collection NA. General remarks can be drawn from the discussion: (i) the fluorescence enhancement factor is not an absolute figure of merit for a given optical antenna design, (ii) the fluorescence lifetime reduction (Purcell factor) must not be confused with the fluorescence count rate enhancement, and (iii) high enhancement factors do not necessarily indicate bright photon sources.

\section{Acknowledgments}

The author is deeply indebted to many at the Fresnel Institute and the Laboratoire des Nanostructures at the Institut de Science et d'Ingénierie Supramoléculaires. He would like to thank Hervé Rigneault, Pierre-François Lenne, and Thomas Ebbesen for stimulating the early stages of this work, Oussama Mahboub and Eloïse Devaux for fabricating the sample presented in Figure 1, and Heykel Aouani for performing the measurements. The author also would like to acknowledge stimulating discussions with Steve Blair, Davy Gérard, Nicolas Bonod, Brian Stout, and Evgeny Popov. To Nicolas, he owes a special thank for stimulating this work consecutively to the Second Summer School on Plasmonics held on Porquerolles in Fall 2011. Lastly, he would like to thank Didier Wampas for inspiring part of this work.The research leading to these results has received funding from the Agence Nationale de la Recherche, Project ANR-11-BS10002 TWINS. The research has been conducted in the scope of the CNRS-Weizmann NaBi European associated laboratory.

\section{References}

[1] L. Novotny and N. H. van Hulst, "Antennas for light," Nature Photonics, vol. 5, pp. 83-90, 2011.
[2] P. Bharadwaj, B. Deutsch, and L. Novotny, "Optical antennas," Advances in Optics and Photonics, vol. 1, pp. 438-483, 2009.

[3] J. A. Schuller, E. S. Barnard, W. Cai, Y. C. Jun, J. S. White, and M. L. Brongersma, "Plasmonics for extreme light concentration and manipulation," Nature Materials, vol. 9, no. 3, pp. 193-204, 2010.

[4] P. Anger, P. Bharadwaj, and L. Novotny, "Enhancement and quenching of single-molecule fluorescence," Physical Review Letters, vol. 96, no. 11, Article ID 113002, 4 pages, 2006.

[5] S. Kühn, U. Håkanson, L. Rogobete, and V. Sandoghdar, "Enhancement of single-molecule fluorescence using a gold nanoparticle as an optical nanoantenna," Physical Review Letters, vol. 97, no. 1, Article ID 017402, 2006.

[6] O. L. Muskens, V. Giannini, J. A. Sánchez-Gil, and J. Gómez Rivas, "Strong enhancement of the radiative decay rate of emitters by single plasmonic nanoantennas," Nano Letters, vol. 7, no. 9, pp. 2871-2875, 2007.

[7] A. Kinkhabwala, Z. Yu, S. Fan, Y. Avlasevich, K. Müllen, and W. E. Moerner, "Large single-molecule fluorescence enhancements produced by a bowtie nanoantenna," Nature Photonics, vol. 3, no. 11, pp. 654-657, 2009.

[8] Y. Fu and J. R. Lakowicz, "Modification of single molecule fluorescence near metallic nanostructures," Laser and Photonics Reviews, vol. 3, no. 1-2, pp. 221-232, 2009.

[9] M. Ringler, A. Schwemer, M. Wunderlich et al., "Shaping emission spectra of fluorescent molecules with single plasmonic nanoresonators," Physical Review Letters, vol. 100, no. 20, Article ID 203002, 2008.

[10] G. Vecchi, V. Giannini, and J. Gómez Rivas, "Shaping the fluorescent emission by lattice resonances in plasmonic crystals of nanoantennas," Physical Review Letters, vol. 102, no. 14, Article ID 146807, 2009.

[11] R. J. Moerland, T. H. Taminiau, L. Novotny, N. F. Van Hülst, and L. Kuipers, "Reversible polarization control of single photon emission,” Nano Letters, vol. 8, no. 2, pp. 606-610, 2008.

[12] H. Gersen, M. F. García-Parajó, L. Novotny, J. A. Veerman, L. Kuipers, and N. F. Van Hulst, "Influencing the angular emission of a single molecule," Physical Review Letters, vol. 85, no. 25, pp. 5312-5315, 2000.

[13] T. H. Taminiau, F. D. Stefani, F. B. Segerink, and N. F. Van Hulst, "Optical antennas direct single-molecule emission," Nature Photonics, vol. 2, no. 4, pp. 234-237, 2008.

[14] S. Kühn, G. Mori, M. Agio, and V. Sandoghdar, "Modification of single molecule fluorescence close to a nanostructure: radiation pattern, spontaneous emission and quenching," Molecular Physics, vol. 106, no. 7, pp. 893-908, 2008.

[15] A. G. Curto, G. Volpe, T. H. Taminiau, M. P. Kreuzer, R. Quidant, and N. F. Van Hulst, "Unidirectional emission of a quantum dot coupled to a nanoantenna," Science, vol. 329, no. 5994, pp. 930-933, 2010.

[16] T. Shegai, V. D. Miljković, K. Bao et al., "Unidirectional broadband light emission from supported plasmonic nanowires," Nano Letters, vol. 11, no. 2, pp. 706-711, 2011.

[17] J. Zhang, Y. Fu, M. H. Chowdhury, and J. R. Lakowicz, "Metal-enhanced single-molecule fluorescence on silver particle monomer and dimer: coupling effect between metal particles," Nano Letters, vol. 7, no. 7, pp. 2101-2107, 2007.

[18] S. Gerber, F. Reil, U. Hohenester, T. Schlagenhaufen, J. R. Krenn, and A. Leitner, "Tailoring light emission properties of fluorophores by coupling to resonance-tuned metallic nanostructures," Physical Review B, vol. 75, no. 7, Article ID 073404, 2007. 
[19] Y. Zhang, K. Aslan, M. J. R. Previte, and C. D. Geddes, "Metalenhanced fluorescence: surface plasmons can radiate a fluorophore's structured emission," Applied Physics Letters, vol. 90, no. 5, Article ID 053107, 2007.

[20] G. Colas des Francs, A. Bouhelier, E. Finot et al., "Fluorescence relaxation in the near-field of a mesoscopic metallic particle: distance dependence and role of plasmon modes," Optics Express, vol. 16, no. 22, pp. 17654-17666, 2008.

[21] F. Tam, G. P. Goodrich, B. R. Johnson, and N. J. Halas, "Plasmonic enhancement of molecular fluorescence," Nano Letters, vol. 7, no. 2, pp. 496-501, 2007.

[22] W. L. Barnes, "Fluorescence near interfaces: the role of photonic mode density," Journal of Modern Optics, vol. 45, no. 4, pp. 661-699, 1998.

[23] J. Enderlein and T. Ruckstuhl, "The efficiency of surfaceplasmon coupled emission for sensitive fluorescence detection," Optics Express, vol. 13, no. 22, pp. 8855-8865, 2005.

[24] L. Rogobete, F. Kaminski, M. Agio, and V. Sandoghdar, "Design of plasmonic nanoantennae for enhancing spontaneous emission," Optics Letters, vol. 32, no. 12, pp. 1623-1625, 2007.

[25] J. S. Biteen, D. Pacifici, N. S. Lewis, and H. A. Atwater, "Enhanced radiative emission rate and quantum efficiency in coupled silicon nanocrystal-nanostructured gold emitters," Nano Letters, vol. 5, no. 9, pp. 1768-1773, 2005.

[26] G. L. Liu, J. Kim, Y. Lu, and L. P. Lee, "Fluorescence enhancement of quantum dots enclosed in $\mathrm{Au}$ nanopockets with subwavelength aperture," Applied Physics Letters, vol. 89, no. 24, Article ID 241118, 2006.

[27] Y. J. Hung, I. I. Smolyaninov, C. C. Davis, and H. C. Wu, "Fluorescence enhancement by surface gratings," Optics Express, vol. 14, no. 22, pp. 10825-10830, 2006.

[28] Y. Liu and S. Blair, "Fluorescence enhancement from an array of subwavelength metal apertures," Optics Letters, vol. 28, no. 7, pp. 507-509, 2003.

[29] D. Gérard, J. Wenger, N. Bonod et al., "Nanoapertureenhanced fluorescence: towards higher detection rates with plasmonic metals," Physical Review B, vol. 77, no. 4, Article ID 045413, 2008.

[30] J. Wenger, D. Gérard, J. Dintinger et al., "Emission and excitation contributions to enhanced single molecule fluorescence by gold nanometric apertures," Optics Express, vol. 16, no. 5, pp. 3008-3020, 2008.

[31] E. Fort and S. Grésillon, "Surface enhanced fluorescence," Journal of Physics D, vol. 41, no. 1, Article ID 013001, 2008.

[32] L. Novotny and B. Hecht, Principles of Nano-Optics, Cambridge University Press, Cambridge, UK, 2006.

[33] H. Aouani, O. Mahboub, N. Bonod et al., "Bright unidirectional fluorescence emission of molecules in a nanoaperture with plasmonic corrugations," Nano Letters, vol. 11, no. 2, pp. 637-644, 2011.

[34] W. L. Barnes, "Comparing experiment and theory in plasmonics," Journal of Optics A, vol. 11, no. 11, Article ID 114002, 2009.

[35] C. Zander, J. Enderlein, and R. A. Keller, Single-Molecule Detection in Solution-Methods and Applications, Wiley-VCH, Berlin, Germany, 2002.

[36] J. R. Lakowicz, Principles of Fluorescence Spectroscopy, Springer, Berlin, Germany, 3rd edition, 2006.

[37] R. Brouri, A. Beveratos, J. P. Poizat, and P. Grangier, "Photon antibunching in the fluorescence of individual color centers in diamond," Optics Letters, vol. 25, no. 17, pp. 1294-1296, 2000.
[38] A. Beveratos, R. Brouri, T. Gacoin, A. Villing, J. P. Poizat, and P. Grangier, "Single photon quantum cryptography," Physical Review Letters, vol. 89, no. 18, Article ID 187901, 4 pages, 2002.

[39] A. F. Koenderink, "On the use of Purcell factors for plasmon antennas," Optics Letters, vol. 35, no. 24, pp. 4208-4210, 2010.

[40] T. H. Taminiau, F. D. Stefani, and N. F. Van Hulst, "Single emitters coupled to plasmonic nano-antennas: angular emission and collection efficiency," New Journal of Physics, vol. 10, Article ID 105005, 2008.

[41] H. Aouani, O. Mahboub, E. Devaux, H. Rigneault, T. W. Ebbesen, and J. Wenger, "Plasmonic antennas for directional sorting of fluorescence emission," Nano Letters, vol. 11, no. 6, pp. 2400-2406, 2011.

[42] K. G. Lee, X. W. Chen, H. Eghlidi et al., "A planar dielectric antenna for directional single-photon emission and nearunity collection efficiency," Nature Photonics, vol. 5, no. 3, pp. 166-169, 2011. 

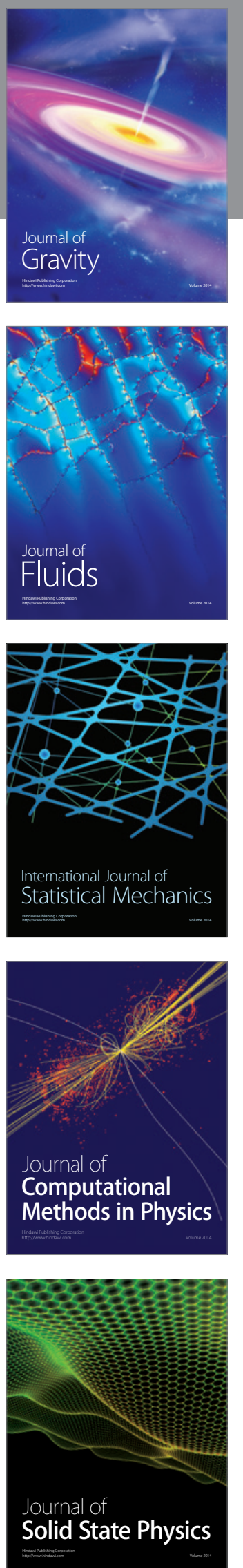

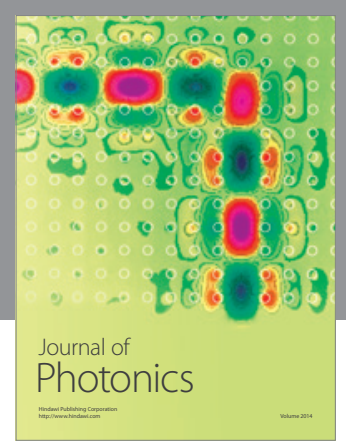

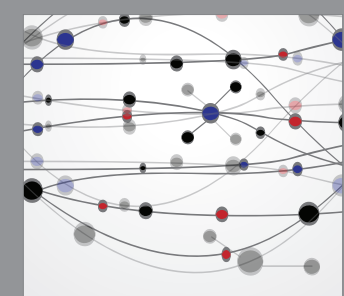

The Scientific World Journal
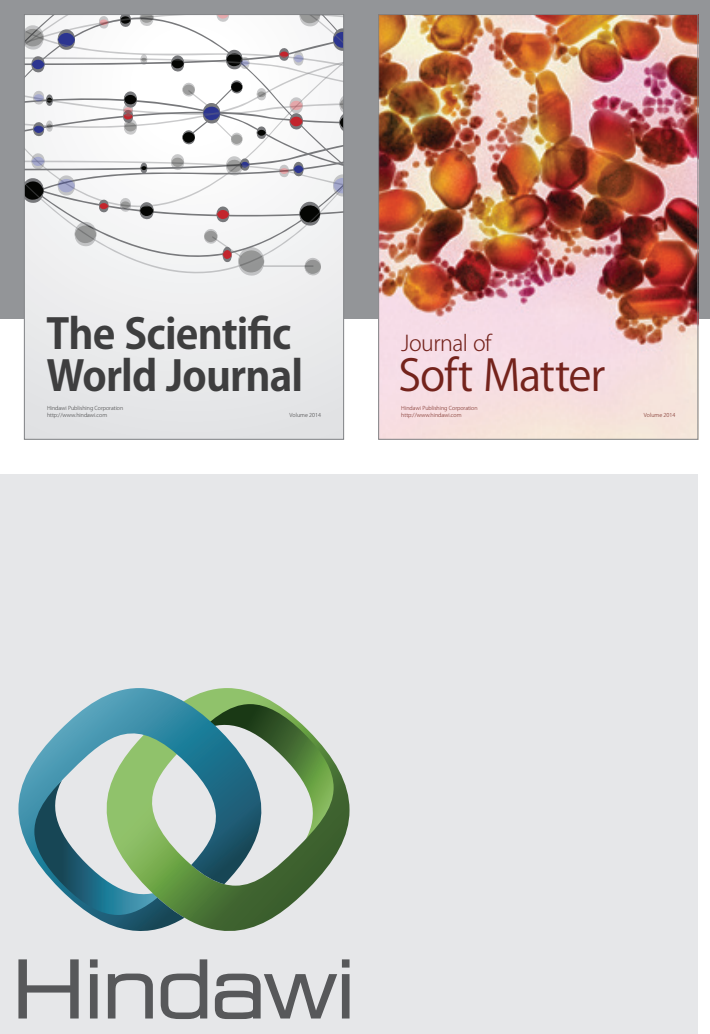

Submit your manuscripts at

http://www.hindawi.com
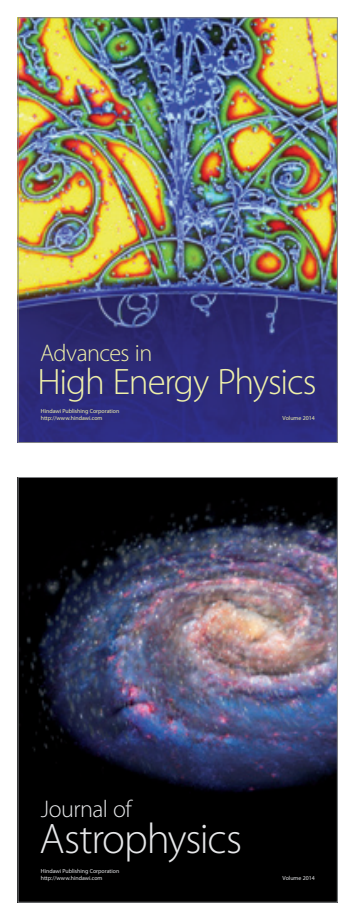
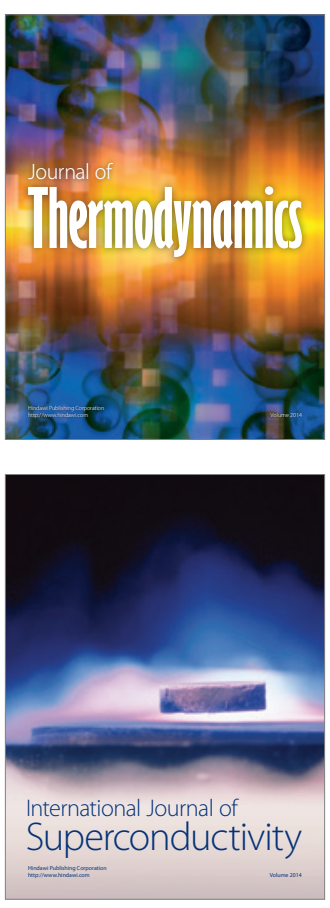
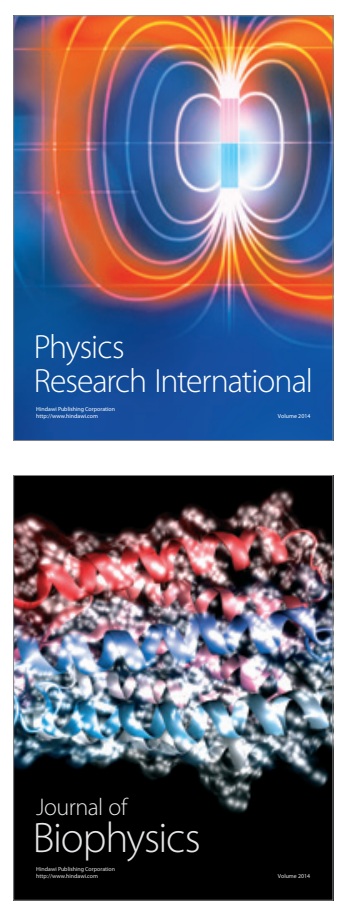
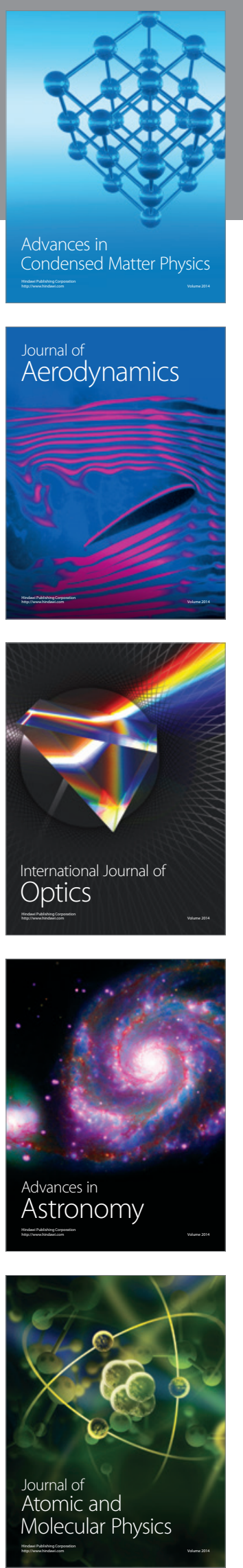